\title{
PHYSICAL METHODS OF FAST REACTIONS INVESTIGATION
}

\author{
Gheorghe Duca ${ }^{a}$, Natalia Secara ${ }^{b *}$, Daniela Duca ${ }^{c}$ \\ ${ }^{a}$ Academy of Science of Moldova, 1 Stefan cel Mare str., MD-2001, Chisinau, Republic of Moldova; \\ ${ }^{b}$ Institute of Chemistry, 3 Academy str., MD-2028, Republic of Moldova, Tel: (0373-22)7297; \\ ${ }^{c}$ Lafayette College, Easton, PA, USA \\ *Corresponding author: E-mail: natalia_secara@yahoo.com
}

\begin{abstract}
This review presents the basic concepts of the methods used for investigation of fast reactions kinetics, such as: flow methods, with particular emphasis on the stopped-flow approach, NMR, ESR, electrochemical methods, with particular emphasis on the time resolved Fourier Transform electrochemical impedance spectroscopy, flash photolysis, and several others. It offers a brief description of fast reactions commonly encountered in chemical systems, providing an insight into the possibilities of performing kinetic investigations of such reaction systems.
\end{abstract}

Keywords: fast reactions kinetics, spectroscopy, relaxation, flow methods.

\section{Introduction}

The outstanding interest in investigating rapid reactions that burst out in the last decades of the last century is nowadays greatly supported by the development of new approaches and methodologies. Modern methods, formed on the basis of classical ones, allow the discovery of precious information about elementary processes in various systems: chemical, biological, environmental, biochemical, real systems or models.

A wide range of physical methods can be applied in order to perform kinetic investigations of rapid reactions, depending on the specificities of the reacting system. Further we will shortly present some of the basic concepts of physical methods of fast reactions investigation and new directions in the field of rapid reactions study.

Fundamental aspects of rapid reactions' research

Usually, fast reactions are divided into three categories, depicted in the figure below:
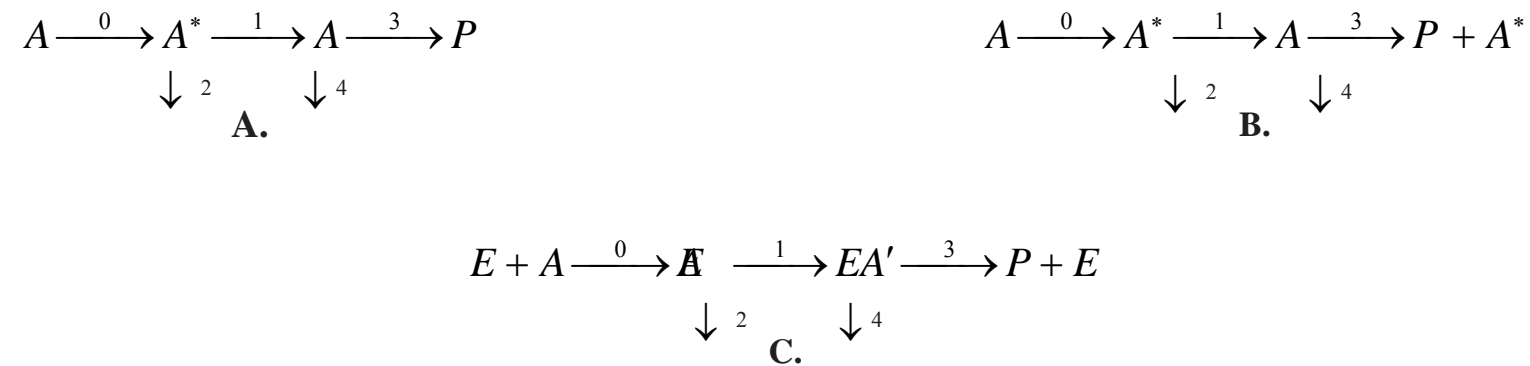

Fig 1. Multi-stage fast reactions.

A. Type I complex reaction - a "simple" multi-stage reaction. B. Type II multi-stage reaction - cyclic, chain reaction.

C. Type III multi-stage reaction - cyclic, catalytic reaction. Where: $\mathrm{A}^{*}$ - active particle; X - intermediate substance; $\mathrm{P}$ reaction product; $\mathrm{E}$ - catalyst; $(0)$ - reaction of reactants activation; $(1,3)$ - reactions that involve an active particle, intermediate substance on the formation of $\mathrm{P} ;(2,4)$ - reactions of $\mathrm{A}^{*}, \mathrm{X}$, not yielding $\mathrm{P}$.

An example of type I complex reaction is the following transformation: $2 \mathrm{Na}+\mathrm{C}_{2} \mathrm{H}_{4} \mathrm{Cl}_{2} \rightarrow 2 \mathrm{NaCl}+\mathrm{C}_{2} \mathrm{H}_{4}$, which involves one-stage reactions steps:

0. $\mathrm{C}_{2} \mathrm{H}_{4} \mathrm{Cl}_{2} \rightarrow \mathrm{C}_{2} \mathrm{H}_{4} \mathrm{Cl}_{2}^{*}$

1. $\mathrm{C}_{2} \mathrm{H}_{4} \mathrm{Cl}_{2}{ }^{*}+\mathrm{Na} \rightarrow \mathrm{NaCl}+\mathrm{C}_{2} \cdot \mathrm{H}_{4} \mathrm{Cl}$

2. $\mathrm{C}_{2} \mathrm{H}_{4} \mathrm{Cl}_{2}^{*} \rightarrow \mathrm{C}_{2} \mathrm{H}_{4} \mathrm{Cl}_{2}$

3. $\mathrm{C}_{2} \cdot \mathrm{H}_{4} \mathrm{Cl}+\mathrm{Na} \rightarrow \mathrm{NaCl}+\mathrm{C}_{2} \mathrm{H}_{4}$

4. $2 \mathrm{C}_{2} \cdot \mathrm{H}_{4} \mathrm{Cl} \rightarrow \mathrm{C}_{4} \mathrm{H}_{8} \mathrm{Cl}_{2}$ 
The reaction rate can be expressed as follows: $\mathrm{w}=\mathrm{d}\left[\mathrm{C}_{2} \mathrm{H}_{4}\right] / \mathrm{dt}=\mathrm{w}_{0}-\mathrm{w}_{2}-2 \mathrm{w}_{4}$.

A wide variety of consecutive and consecutive-parallel reactions as such are known. For instance, photochemical reactions are a significant group of reaction that can be related to this mechanism. No more than one particle of reaction product is formed per one adsorbed light quantum during these reactions.

Cyclic reactions proceed according to type II and type III mechanisms. A particular feature of such reactions is the formation of a particle in addition to the products. This particle then participates in one of the reactions that lead to the formation of the product. There are two types of cyclic transformations: chain and catalytic. It is important that reaction rates of chain transformations are higher than the rate of active particles formation:

$$
\mathrm{w}=\mathrm{dP} / \mathrm{dt}=\mathrm{w}_{0} \mathrm{v},
$$

where $v$ is the chain length, shows how many particles of $\mathrm{P}$ product are formed per active particle which are formed during the activation stage (0). Reactions are known with $v \approx 10^{5}$, but usually $v \approx 10-10^{3}$.

The photolysis of $\mathrm{H}_{2} \mathrm{O}_{2}$ in aqueous solutions is one of the many examples of chain transformations:

$$
\begin{aligned}
& \text { 0. } \mathrm{H}_{2} \mathrm{O}_{2} \rightarrow \mathrm{H}_{2} \mathrm{O}_{2}^{*} \rightarrow 2 \mathrm{OH}^{*} \\
& \text { 2. } \mathrm{H}_{2} \mathrm{O}_{2}^{*} \rightarrow \mathrm{H}_{2} \mathrm{O}_{2} \\
& \text { 1. } \mathrm{OH} \bullet+\mathrm{H}_{2} \mathrm{O}_{2} \rightarrow \mathrm{H}_{2} \mathrm{O}+\mathrm{HO}_{2} \bullet \\
& \text { 3. } \mathrm{HO}_{2} \bullet+\mathrm{H}_{2} \mathrm{O}_{2} \rightarrow \mathrm{H}_{2} \mathrm{O}+\mathrm{O}_{2}+\mathrm{OH} \bullet \\
& \text { 4. } \mathrm{HO}_{2} \bullet+\mathrm{HO}_{2} \bullet \rightarrow \mathrm{H}_{2} \mathrm{O}_{2}+\mathrm{O}_{2} .
\end{aligned}
$$

For the wide majority of the studied chain processes, the active particles are atoms and free radicals, and the processes are called radical-chain. The lifetime of active particles such as $\mathrm{OH} \bullet, \mathrm{CH}_{3} \bullet$, etc. is short due to their high reactivity, and low concentrations, compared to the typical reaction time and reagents' concentrations. The nonstationary period, where the concentrations of active particles increase from zero to stationary values, is usually around milliseconds. The method of quasi-stationary concentrations can be applied for most reactions, with regard to active particles.

The second type of cyclic reactions is represented by catalytic reactions, when the activation of the reagent proceeds not as a result of thermal, photochemical or other influence, but as a result of its interaction with the introduced substance-catalyst [1]. Due to the fact that the cycle is closed on the link preceding the activation stage, the rate of a catalytic reaction cannot be higher than the activation rate of the interaction between $\mathrm{A}$ and $\mathrm{E}$.

Catalytic reactions are similar to chain reactions, considering their cyclic mechanism, and are similar to "simple" fast-reactions of type I for the reaction rate does not exceed the rate of the reagents activation on their interaction with the catalyst.

The limiting stage for catalytic cycle reactions is the share of the catalyst present in the (EA)' complex; the initial activation of the reagent - EA complex formation - is seldom a limiting stage, in contrast to radical-chain reactions, where the rate of the process depends on the initial formation of atoms or radicals from the reagent molecules.

Taking into account that the most important fast reactions with relevance to chemical and biological systems take place in liquid media, there are three general factors that are to be considered in case of investigating the reaction rates in solution $[2,3]$ :

- The rate at which initially separated reactant molecules come together and become neighbours, i.e. the rate of encounters.

- The time that two reactants spend as neighbours before moving away from each other, i.e. the duration of an encounter. During this time, the reactants may collide with each other hundreds of times.

- The requirement of energy and orientation which two neighbouring reactant molecules must satisfy in order to react.

Any one of these three factors may dominate in governing the reaction rate.

Many chemical reactions occur as soon as the reactant molecules come together and thus, for these fast reactions, the activation energy and orientation requirements are negligible. The reaction rate is limited only by the first factor (i.e., the rate at which encounters occur), which makes it diffusion-controlled.

A simple mechanism for these diffusion controlled reactions may be given by the following kinetic scheme:

$$
\mathrm{A}+\mathrm{B} \underset{k_{-d}}{\stackrel{k_{d}}{\rightleftharpoons}}(\mathrm{AB}) \stackrel{k_{r}}{\rightarrow} \text { products }
$$

where $k_{d}$ is the rate constant for diffusion of A and B towards each other, $k_{-d}$ is the rate constant for diffusion of A and $\mathrm{B}$ away from each other, and $k_{c}$ is the rate constant for the conversion of the complex (AB) to product $\mathrm{R}$ (i.e., the rate constant with out diffusive effects). 
Using the Steady-State Approximation on (AB):

$$
\frac{d[(A B)]}{d t}=0=k_{d}[A][B]-k_{-d}[(A B)]-k_{c}[(A B)] \Rightarrow[(A B)]\left(k_{-d}+k_{c}\right)=k_{d}[A][B] ;[(A B)]=\frac{k_{d}[A][B]}{k_{-d}+k_{c}}
$$

Thus, the reaction velocity is given by the equation: $r=k_{r}[(A B)]=\frac{k_{r} k_{d}[A][B]}{k_{-d}+k_{r}}$

Thus, the rate constant of the bimolecular reaction $\mathrm{A}+\mathrm{B} \stackrel{k_{2}}{\longrightarrow}$ products, is given by : $k_{2}=\frac{r}{[A][B]}=\frac{k_{r} k_{d}}{k_{-d}+k_{r}}$

In this case, two limiting cases should be considered:

(i) diffusion-controlled reaction: $\mathrm{k}_{\mathrm{r}} \gg \mathrm{k}_{-\mathrm{d}}$, separation of $\mathrm{A}$ and $\mathrm{B}$ is relatively difficult, e.g., the solvent has a large viscosity $\eta$, or the reaction has a small activation energy, so the rate-determining step is the diffusive approach of the reactants; once they are in the solvent reaction cage, reaction is assured, $\mathrm{k}_{2}=\mathrm{k}_{\mathrm{d}}$ (where for aqueous solutions at room temperature, $\mathrm{k}_{\mathrm{d}} \approx 10^{9}-10^{10} \mathrm{M}^{-1} \mathrm{~s}^{-1}$, and a value of $\mathrm{k}_{\mathrm{d}}>10^{9} \mathrm{M}^{-1} \mathrm{~s}^{-1}$ usually indicates that the aqueous solution reaction is diffusion controlled).

(ii) activation-controlled reaction: $\mathrm{k}_{\mathrm{r}}$ « $\mathrm{k}_{-\mathrm{d}}$, reactions with large activation energies, $\mathrm{E}_{\mathrm{a}} \geq 20 \mathrm{~kJ} \mathrm{~mol}^{-1}$ for reactions in water $k_{2}=k_{r} \frac{k_{d}}{k_{-d}}$

where the second factor is the equilibrium constant $\mathrm{K}_{\mathrm{AB}}$ for the formation of the encounter pair; thus $\mathrm{k}_{2}=\mathrm{k}_{\mathrm{r}} \mathrm{K}_{\mathrm{AB}}$, and the reaction rate is determined by the equilibrium concentration of encounter pairs and the rate of passage over the activation energy barrier.

One way to assess the validity of the assumption of fast chemistry is to estimate the Damkohler number [3], an important dimensionless parameter that quantifies whether a process is kinetically or diffusion controlled. Damkohler number is defined as $\mathrm{Da} \equiv \tau_{\text {mix }} / \tau_{\text {react }}$. Here, $\tau_{\text {mix }}$ is a characteristic mixing time for the system, while $\tau_{\text {reac }}$ is a characteristic time for the chemical reaction. If the Damkohler number has a value much larger than unity, the process is diffusionally controlled, while a value much lower than unity indicates a kinetically controlled process.

Another factor which affects the rate of reactions in solution involves the effects of solvent molecules on the motions of reactant molecules.

Once two reactant molecules diffuse together, their first collision may not satisfy the energy and orientation requirements for reaction. The surrounding solvent molecules create a "solvent cage" which inhibits the separation of the reactant molecules.

This solvent "cage effect" provides the opportunity for the molecules to undergo many more collisions before finally either reacting or diffusing away from each other.

The duration of such an encounter may be $10^{-10} \mathrm{~s}$, during which the reactant molecules may collide hundreds of times. The cage effect tends to increase the reaction rate by increasing the opportunity for reactant molecules to find the required energy and/or orientation to react during the caged encounter.

However, there are a large number of reactions that are initiated by the formation of a reactive species in the investigated solution, rather than by mixing the reagents. In the case of such chemical reactions it has to be taken into consideration that the duration of the initiation process has to be a lot shorter than the reaction time and it has to be performed by techniques that would not alter the solution's homogeneity.

The main physical methods that are currently used in the investigation of rapid reactions are reviewed below.

\section{Basic concepts of the methods that are used to study fast reactions}

\section{Flow-based Methods}

Flow analysis comprises all analytical methods that are based on the introduction and processing of test samples in flowing media. A primary classification can be based on two aspects [4]:

- the way the test portion is introduced, i. e. continuously or intermittently/discretely,

- the basic character of the flowing media, i.e. either segmented, unsegmented or monosegmented where segmentation is primarily considered as being applied for the purpose of preventing intermixing of successive analyte zones.

However, among all the flow methods used for the study of fast reactions the most popular seems to be the stopped-flow method. Due to the short analysis time and feasibility, flow based systems have become one of the most 
powerful analytical tools used for studying fast reactions. This can also be explained by the possibility of employing most various detection systems.

The apparatus [5] is made of 2 reservoir syringes that are loaded with the desired species. A pneumatic syringe push will drive the reactant species to the mixing tube or jet. The syringe drive could be positioned either horizontally or vertically. The mixed solutions then pass to the measuring cell or the observation chamber where the data is collected. If the reaction involves three reactants, a modification to have 3 drive syringes and two mixing tubes is required (KinTek Corporation, 2007). The optical path can be either along this tube or perpendicular to it. Finally, the reaction mixture fills the stopping syringe which triggers the start of data acquisition. The lifetime of the reactions that can be analyzed using the stopped-flow apparatus is limited by the time it takes the solution to fill in the measuring cell, also know as the "dead time".

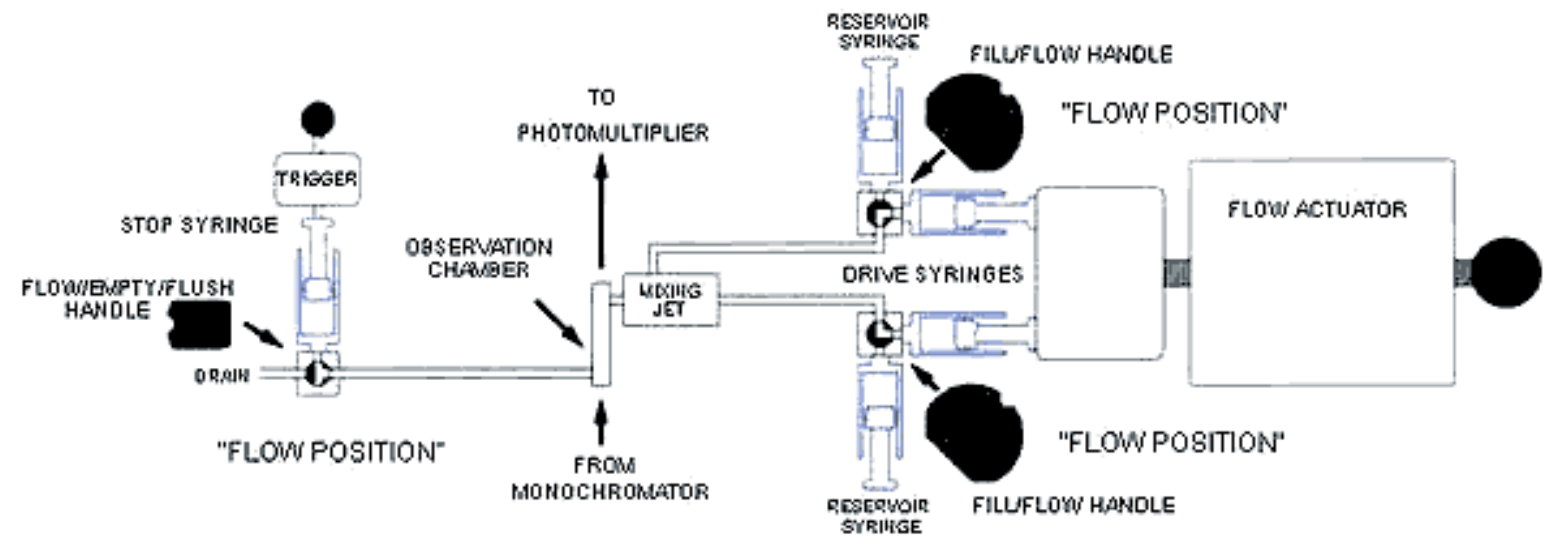

Fig. 2. Diagram of a stopped-flow apparatus

There are three main ways to compute the kinetic parameters via the stopped-flow: steady-state approach, taking the concentration gradient into account, and standard second order treatment assuming the conditions mentioned earlier. Dunn and Rorabacher [6] determined that all three are reliable in the analysis of second order reactions. The standard treatment can be used when the rate constant is on the order of (1-4) x $10^{6} \mathrm{M}^{-1} \mathrm{~s}^{-1}$. However, if the second order reaction half-life is less than the dead time, then the steady state approach should be used to check the viability of results. In the case when the values of the rate constant obtained by the standard approach and that obtained when considering the concentration gradient are significantly different, the steady state should be considered as the better approach to determining the parameter. In order to assess the validity of this result, different initial concentrations of the reactants can be used via an iterative approach until a consistency in the rate constant is obtained [6].

The stopped-flow method has been extensively applied in scientific investigations, such as: studying the dimertetramer equilibria of oxyhemoglobins and the kinetics of association of unligated dimmers [7], determination of nitrite in natural and drinking-water [8], fluorimetric determination of uric acid in serum and urine samples [9], study of the mechanism of bacteriophage T7 RNA polymerase binding to its promoter DNA [10], spectroscopic detection and kinetic analysis of aromatic amine cation radicals [11], activation of $\alpha$-chymotrypsin [12], complex formation processes $[13,14]$, redox catalytic processes $[15,16]$, and many others.

Stopped-flow mixing coupled with time-resolved Fourier transform infrared (FT-IR) spectroscopy represents a new experimental approach to explore protein folding events, with one specific advantage - the ability to monitor directly the kinetics of processes involving $\beta$-sheet structures [17], which is exceptionally difficult to do with other techniques. The temperature-jump/stopped-flow method combined with the application of structure specific fluorescence signals provides novel opportunities to study the stability of certain regions of enzymes and identify the unfolding-initiating regions of proteins.

\section{NMR methods}

NMR methods have a significant advantage over other methods used for the investigation of fast processes that are not accompanied by veritable chemical reactions.

The NMR methods are largely used for investigation of equilibrated chemical transformations and of exchange processes, which are accompanied by periodic structure modifications, i.e. modifications of nuclei electronic surroundings and nuclei spin-spin interactions. These processes refer to intramolecular transformations (tautomery [18], racemization [19], delayed internal rotation, cycle inversion [20]), as well as to intermolecular exchange chemical reactions and other equilibrated reactions (protons exchange in aqueous solutions of carbonic acids, ammonia, ligand exchange [21], ions recombination, biochemical enzyme-substrate interactions). 


\section{ESR methods}

It is almost impossible to observe the reactive free radicals using direct methods, due to their low stationary concentration. Such methods as photometry, UV-, IR-spectroscopy, radiospectroscopy, allow the registration of the less reactive, rather stable radicals of secondary origin [22]. The electronic spin resonance technique is extremely used in the investigation of mechanisms and kinetic laws of chemical reactions unfolding. This is mainly due to the role played by paramagnetic particles in chemical processes, and by the high sensitivity of the method, as well.

The utilization of ESR coupled with flow methods is very widespread: the reaction mixture is moved rapidly through the cell in the resonator, making possible to maintain concentrations of unstable radical species, high enough and for a time long enough to allow spectra registration. This offers the possibility to monitor intermediate compounds formed during the reaction, as well as the determination of rate constants of individual elementary stages of the process.

\section{IR spectroscopy}

In general, IR spectroscopy is difficult to implement in fast reactions researches [23], but good results can be obtained when using this method in addition to the others (temperature-jump technique, stopped-flow method etc.).

A widespread technique largely applied in the study of rapid reactions is the IR spectroscopy coupled with Fouriertransform (FTIR), which is an interferometric method, as opposed to classic spectrometry where the spectral absorption is scanned. FTIR spectrometers have high sensitivity, but the time-resolved performance is limited by the scan rate, unless step-scan or stroboscopic methods are used for the study of events that can be repetitively photoactivated [24].

Time-resolved infrared absorption spectroscopy is widely used to probe the kinetics of short-lived (down to $\sim 1 \mathrm{~ms}$ ) coordinatively unsaturated organometallic species, thus allowing scientists to deepen their understanding of organometallic reaction mechanisms. IR spectroscopy has the twin advantages that many organometallic complexes have strong absorptions in the infrared, making detection relatively straightforward, and that the IR spectrum of an organometallic complex frequently reveals information about its electronic structure. Also, time-resolved FT-IR difference spectroscopy in combination with molecular biological methods allows a detailed analysis of the molecular reaction mechanism of proteins from the nanosecond time range up to several minutes [25].

\section{UV-VIS spectroscopy}

UV-visible spectroscopic methods are well suited for timeresolved measurements, and these can be made in the subpicosecond range if lasers are used. They suffer from the disadvantage that little structural information is provided [26], although in favourable cases, such as in studies of bacteriorhodopsin, where the intermediates of the photocycle show distinct and accurately interpretable spectral characteristics, these methods have proved very valuable [27]. Among the UV-VIS spectrometric methods, photocolorimetry is currently very popular, especially due to its very sensitive detection limit $\left(10^{-5}-10^{-6} \mathrm{M}\right)$ and a relative error value of $1-2 \%$ for the majority of experiments. Thus, the possibilities of electronic spectra application emerge from their tight correlation with the molecular structure and the character of the interaction of these molecules with the surroundings.

\section{Flash photolysis}

This technique is commonly used for studying fast photochemical reactions. Generally, the pulse width of the light source must be much shorter than the half-time of the chemical reaction. Thus, depending on the rate of the process under investigation, several lamps are available. In the case of moderate rates of reactions (microsecond time scale) flash lamps can be used; a typical flash lamp is the xenon lamp in a standard camera. For very fast reactions, however, the slow decay time of the light emission from a flash lamp covers the progress of the reaction. For faster reactions, specially designed lasers must be used that have pulse widths in the nanosecond range. Using ultra-fast pulsed lasers allows processes in the sub-femtosecond time scale to be studied.

Pulse radiolysis is a radiochemical analogy of the flash photolysis technique, where the sample is excited by a short pulse $\left(10^{-9}-10^{-6} \mathrm{~s}\right)$ of ionizing radiation (usually high energy electrons). Instruments used in pulsed radiolysis can be classified according to their characteristic time, which can be micro-, nano- or picoseconds.

Pulse radiolysis method offers important information on the spectra and reactivities of electrons solvated in polar liquids, reactivities of hydroxyl radicals in aqueous solutions, spectra and kinetic behaviour of free radicals in solutions (organic and inorganic), optic spectra of the singlet states of the anion-radicals of aromatic compounds etc.

\section{Relaxation techniques}

In the methods that use relaxation phenomena, a system in equilibrium is perturbed by a sudden change of external parameters, such as temperature, pressure or the electric field intensity. The kinetic study of the instauration of the new equilibrium delivers information about the chemical reactions that take place in the system. Due to the fact 
that relaxation methods don't require rapid mixing of reagents, the characteristic time is determined only by the rate at which the equilibrium is perturbed. This allows performing kinetic investigations of reactions that take place in seconds to $10^{-10} \mathrm{~s}$. Besides the large time interval, relaxation methods facilitate the analysis of complex reaction systems [28]. As the variations of external parameters are always quite small, the equations that describe the kinetic behaviour of the system can be easily linearized. The process of relaxation of the system to the new equilibrium is described by a set of relaxation times, related to the mechanism of the reaction.

Methods of equilibrium perturbation, used for the estimation of relaxation times comprise:

- methods of discrete perturbation: temperature-jump technique, pressure-jump technique and electric field jump technique. The methods used for monitoring and registration of concentration variations have to possess high specificity and sensibility (spectrophotometry, fluorimetry, polarimetry, conductometry).

- methods of periodic perturbation: sound wave absorption technique, dielectric losses technique. In these methods, the chemical relaxation processes lead to a phase shift between the external parameters variations and parameters that describe the relaxing system. The amplitude of this shift, or the energy dissipation that accompanies it, are determined by the reaction mechanism.

\section{Electrochemical methods}

Major electroanalytical techniques that can be successfully applied if the investigated compound possesses a certain electroactivity, are conductometry, voltammetry (including polarography, triangular wave voltammetry, AC voltammetry, differential pulse voltammetry, and pulse voltammetry), potentiometry and amperometry. The choice depends on the species under investigation.

A powerful tool for investigating electrochemical reactions is the electrochemical impedance spectroscopy. Still, its application is generally limited to certain experimental systems where the applied D.C. voltage must be kept fixed during the time (at least several minutes in most cases) when an impedance spectrum is recorded, and this makes it quite difficult to study transient systems. Apart from this limitation, it is also difficult in traditional EIS to distinguish between adsorption and desorption (or anodic and cathodic) processes. The time resolved technique of FT-EIS expands the capabilities of EIS beyond these constraints [29]. FT-EIS can provide detailed kinetic information about surface reactions at solid-liquid interfaces and thus, can considerably help to understand the mechanisms of multi-step reactions in multi-component solutions [30]. FT-EIS is particularly useful for studying kinetically controlled time dependent electrochemical systems. These include: oxidation of organic molecules (for fuel cell studies), electrocatalysis, double layer characterization, electrodeposition studies, characterization of bio-electrochemical reactions, metal dissolution reactions (relevant for chemical mechanical and electrochemical mechanical planarization), electrochemistry of ionic liquids, real time monitoring of corrosion reactions.

There is a wide variety of methods available for those who deal with fast reactions kinetics, and new possibilities arise every day for the study of chemical and biochemical processes that have been a mystery until not so long ago.

\section{New directions and approaches in the investigation of fast reactions: DNA related reactions}

Oxygen is essential for life. However, active forms of oxygen such as hydroxyl and superoxide radicals, hydrogen peroxide and singlet oxygen arise as by-products and intermediates of aerobic metabolism and during oxidative stress [31]. Directly or indirectly, these chemical species of oxygen, as well as reactive species of nitrogen, can transiently or permanently damage nucleic acids, lipids, and proteins [32].

Aerobic organisms are normally protected by a defence system against oxidative damage, which comprises various compounds with different functions, including enzymes (e.g. catalase, glutathione peroxidase, glutathione transferase, superoxide dismutase) and the sequestration of metals (e.g. iron, copper) by chelating agents. In addition, there are several hydrophilic and lipophilic compounds, such as vitamin $\mathrm{C}$ and vitamin $\mathrm{E}$, which can act by scavenging or suppressing the generation of free radicals. These are the reasons for the impressive number of papers that deal with the investigation of fast reaction related to the factors that decrease the defence against reactive damaging species.

A number of investigations have revealed that vitamin $C$ and $E$ levels are decreased by exposure to ethanol. Recent research has suggested that ethanol may exert its cell toxicity via DNA damage, possibly via the generation of ROS arising from microsomal NADPH-dependent electron transfer and the oxidation of the ethanol metabolite, acetaldehyde [33]. There was a significant increase in the number of in vivo single-strand breaks in the DNA of rat brain cells after an acute dose of ethanol [34]; ethanol combined with acetaldehyde induced cleavage of DNA in rat hepatocytes [35] and ethanol-induced ${ }^{\mathrm{DNA}}$ fragmentation and cell death was also observed in mouse thymocytes [36]. Ethanol alone, however, did not induce in vitro ${ }^{\text {DNA }}$ strand breaks in human lymphocytes, whereas acetaldehyde induced both single- and doublestrand breaks (30), indicating that ethanol may induce DNA strand breaks via its primary metabolite. The spin trapping technique has been used to demonstrate the increased generation of 1-hydroxyethyl radical both in vitro and in vivo [37]. P.Navasumrit and co-workers have used these procedures to follow the effects of acute and chronic doses of ethanol on the generation of free radicals and we have further attempted to correlate free radical generation with the evolution of 
DNA strand breaks using single electrophoresis. Results of these studies indicate that ethanol-induced DNA damage is mediated by free radicals and that both can be significantly inhibited by prior treatment with antioxidants.

Cellular DNA is subjected to the action of reactive oxygen species during the entire lifecycle of the cell.

The most common purine base redox lesions in vivo are 8-oxoguanine (8-oxoG), 8-oxoadenine, 4,5-diamino5-formamidopyrimidine (FapyAde) (derived from adenine) and 2,6-diamino-4-hydroxy-5-formamidopyrimidine (FapyGua) (derived from guanine) [38].

A large number of scientific work $[39,40,41]$ is related to the investigation of the process of incorporation of inappropriate nucleotides by DNA polymerases opposite these lesions, which results in a considerable mutagenicity. However, there are enzymatic systems that counteract this damage.

For example, it is known that human 8-oxoguanine-DNA glycosylase (hOgg1) excises 8-oxo-7,8-dihydroguanine from damaged DNA. Recently, Kuznetsov et al. [42] have performed a kinetic analysis of hOgg1 mechanism using stopped-flow and enzyme fluorescence monitoring, and proposed a kinetic scheme for hOgg1 processing an 8-oxoG:Ccontaining substrate. The authors report that the mechanism consists of at least three fast equilibrium steps followed by two slow, irreversible steps and another equilibrium step. The second irreversible step was rate-limiting overall. By comparing data from Ogg1 intrinsic fluorescence traces and from accumulation of products of different types, the irreversible steps were attributed to two main chemical steps of the Ogg1-catalyzed reaction: cleavage of the $\mathrm{N}$-glycosidic bond of the damaged nucleotide and B-elimination of its 3'-phosphate. The fast equilibrium steps were attributed to enzyme conformational changes during the recognition of 8-oxoG, and the final equilibrium, to binding of the reaction product by the enzyme.

Analogically, uracil DNA glycosylase (UNG) is responsible for the removal of uracil from DNA by hydrolysis of the N-glycosidic bond that links the base to the deoxyribose backbone [43]. Despite the significant number of scientific papers discussing the possible mechanisms employed at the protein/DNA interface, there are considerable differences in interpretation and there remains a lack of information regarding the dynamics of interactions at the protein-DNA interface, which are the key to understanding the biophysical basis of base flipping. Bellamy et al. [44] have performed a rapid kinetic analysis with DNA substrates of different sequence in order to examine the dynamics of the protein-DNA interface in base flipping by UNG. The stopped-flow analysis (fluorescence and anisotropy) was used to obtain an insight into the binding of substrate and the formation of the activated complex, and quench-flow analysis allowed the investigation of the chemical cleavage step.

Lately, ESR methods have gained a huge popularity in the investigation of radicals formed during the oxidation of DNA, which has implications in mutations, strand breaks and cell death $[45,46]$. The interactions among radical species largely depend on both the amount and location of radicals generated. Therefore, the detection and simultaneous mapping of individual radical species generated in vivo are both essential for understanding the function of radical formation in vivo [47].

Thus, in vivo ESR spectroscopy allows studying and understanding the redox reactions in physiological phenomena [48,49] and in oxidative injuries, such as hypoxia-hyperoxia [50], iron overload [51], and selenium deficiency [52]. The combination of in vivo ESR spectroscopy with an imaging technique allows 2D or 3D imaging of radicals in living organisms [53]. However, biological systems are very complex and multiple radical species coexist within an organ; therefore, it is critical that the individual radical species be visualized separately. Ken-ichiro Matsumoto and Hideo Utsumi have developed a method of separable ESR-CT (electron spin resonance-computed tomography) imaging for multiple radical species, and applied it to imaging of $\cdot \mathrm{OH}$ and $\cdot \mathrm{NO}$. The algorithm was improved by combining filtered back-projection with a modified algebraic reconstruction technique to enhance accuracy and shorten calculation time. This ESR-CT technique was combined with L-band ESR spectroscopy and applied to the separative imaging of $\cdot \mathrm{OH}$ and $\cdot \mathrm{NO}$, which were spin trapped with 5,5-dimethyl-1-pyrroline- $N$-oxide(DMPO) and Fe ${ }^{2+}-N$-methyl-

D-glucamine dithiocarbamate complex, respectively. The present results strongly suggest that this technique would be further applicable to in vivo investigation of other free radical reactions. This separable ESR-CT imaging technique might become one of the most powerful diagnosis techniques [53].

\section{Closing notes}

In this review, it has been shown that various techniques exist that can be applied for studying fast chemical reactions, which can be correlated to the specificity of the reaction under investigation. Whether the reaction is equilibrated or irreversible, whether the species investigated are unstable radicals, or possesses electroactivity, or display luminescence, there is always a possibility to perform scientific investigations by coupling several physical methods.

This article offers a brief review of physical methods commonly used to study rapid reactions. The truly vertiginous development of various techniques (mostly hyphenated methods) for investigating fast reactions has enabled scientists to reveal complex laws of many chemical reactions, and this progress is so unequivocal that it seems only to be limited by scientists' creativity and ingenuity. 


\section{References}

[1] Sychev A., Duca, Gh. Fundamental and Applied Aspects of Homogeneous Catalysis with Metal Complexes. Chisinau, Moldova State University Publ., 2002.

[2] Isac V., Onu A., Tudoreanu C., Nemtoi G., Physical chemistry, Chisinau, Stiinta Publ., 1995, Robert J. Kee, Chemically reacting flow. Theory and practice, New Jersey: John Wiley \& Sons, 2003.

[3] Robert J. Kee, Chemically reacting flow. Theory and practice, New Jersey: John Wiley \& Sons, 2003.

[4] Pure \& Appl. Chem., Vol.66, No 12, 1994, pp. 2493-2500.

[5] “U.S.A. Stopped-Flow: Block Diagrams". Retrieved from http://olisweb.com.

[6] Dunn B. C, Meagher N. E., Rorabacher D. (1996) J. Phys. Chem., 100, 16925-16933.

[7] Berjis M, Sharma VS, Double mixing stopped-flow method for the study of equilibria and kinetics of dimertetramer association of hemoglobins: studies on Hb Carp, $\mathrm{Hb}$ A, and Hb Rothschild, Anal Biochem. 1991 Aug 1;196(2):223-8.

[8] Rui-Yong WANG, Xiang GAO and Ying-Tang LU, Anal. Sci., Vol. 22, 2006, p. 299.

[9] D. Perez-Bendito, A. Gomez-Hens, M.C. Gutierrez and S. Anton, Nonenzymatic stopped-flow fluorimetric method for direct determination of uric acid in serum and urine, Clinical Chemistry 35: 1989, p. 230-233.

[10] Yiping Jia, Amarendra Kumar and Smita S. Patel, Equilibrium and Stopped-flow Kinetic Studies of Interaction between T7 RNA Polymerase and Its Promoters Measured by Protein and 2-Aminopurine Fluorescence Changes, J. Biol. Chem., vol.271, No. 48, 1996, pp. 30451-30458.

[11] Oyama Munetaka, Spectroscopic Detection and Kinetic Analysis of Aromatic Amine Cation Radicals Using an Electron Transfer Stopped-Flow Method, Erekutoro Oganikku Kemisutori Toronkai Koen Yoshishu, V.25, 2001, pp.71-72.

[12] Gert Verheyden, Janka Matrai, Guido Volckaert and Yves Engelborghs, A fluorescence stopped-flow kinetic study of the conformational activation of $\alpha$-chymotrypsin and several mutants, Protein Science, 2004, vol 13, p. 2533-2540.

[13] Duca, Gh., Scutaru, Yu., Sychev A. Kinetics of Interaction of Iodine with Dihydroxyfumaric Acid, J. of Physical Chem., vol.61, 1987, no 8, p.2266-2268.

[14] Duca, Gh., Travin, S., Purmali, A. Catalytic Condensation of Epycatechol in the Presence of Iron Ion, J. of Physical Chem., 1989, vol.63, no 12, p.3214-3220.

[15] Duca, Gh., Scurlatov, Yu., Sychev, A. Redox Catalysis and Ecological Chemistry. - Chisinau, Moldova: CEUSM, 2002, p. 316.

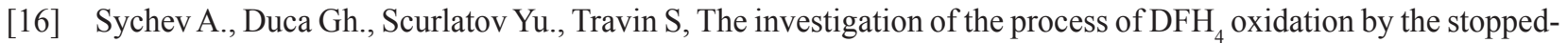
flow method, Phys. and mathem. methods in coord. chem. Abstracts of the $7^{\text {th }}$ congress, Chisinau, 1980, p. 32-33.

[17] Heinz Fabian and Dieter Naumann, Methods to study protein folding by stopped-flow FT-IR, Methods, 2004, Vol. 34, issue 1, p. 28-40.

[18] Dieckmann H., Kreuzig R., Bahadir M., Significance of keto-enol-tautomery in the analysis of 1,2,4-triazinone metabolites, Fresenius' j. anal. chem., vol. 348, n¹1, pp. 749-753 , 1994.

[19] Philip J. Bailey et al., Barriers to Racemization in $\mathrm{C}_{3}$-Symmetric Complexes Containing the Hydrotris(2mercapto-1-ethylimidazolyl)borate ( $\mathrm{Tm}^{\mathrm{Et}}$ ) Ligand, Inorg. Chem., 44 (24), 8884 -8898, 2005.

[20] Georgii E. Salnikov, Alexander M. Genaev and Victor I. Mamatyuk, Unusually strong temperature dependence of ${ }^{13} \mathrm{C}$ chemical shifts and degenerate conformational exchange in cyclobutenyl carbocations, Mendeleev Commun., 2003, 13, 48 - 49.

[21] von Ahsen B, Bach C, Balzer G, Bley B, Bodenbinder M, Hägele G, Willner H, Aubke F., Dynamic 13C NMR studies of ligand exchange in linear (d10) silver(I) and gold(I) and square-planar (d8) rhodium(I) homoleptic metal carbonyl cations in superacidic media, Magn Reson Chem. 2005 Jul;43(7):520-7.

[22] Gh. Duca, Free radicals in natural water, in Free radicals in biology and Environment, edited by F. Minisci, Vol.27, p. 475.

[23] Andrew J. White, Kevin Drabble and Christopher W. Wharton, A stopped-flow apparatus for infrared spectroscopy of aqueous solutions, Biochem. J., 1995, vol. 306, p. 843-849.

[24] Gerwert, K. (1993) Curr. Opin. Struct. Biol. 3, 769-773

[25] Klaus Gerwert, Molecular Reaction Mechanisms of Proteins Monitored by Time-resolved FT-IR Difference Spectroscopy, John Wiley \& Sons Ltd, Chichester, 2002.

[26] Andrew J. White, Kevin Drabble and Christopher W. Wharton, A stopped-flow apparatus for infrared spectroscopy of aqueous solutions, Biochem. J., 1995, vol. 306, p. 843-849.

[27] Doig, S. J., Reid, P. J. and Mathies, R. A. (1991) J. Phys. Chem. 95, 6372-6379.

[28] G. Hammes, Investigation of rates and mechanisms of reactions, "Mir", Moscow, 1977.

[29] J. E. Garland, C. M. Pettit and D. Roy, Analysis of experimental constraints and variables for time resolved detection of Fourier transform electrochemical impedance spectra, Electrochimica Acta 49 (2004) 2623-2635. 
[30] J.E. Garland, K. A. Assiongbon, C.M. Pettit, S.B. Emery and D. Roy, "Kinetic analysis of Electrosorption using Fast Fourier Transform Electrochemical Impedance spectroscopy: Underpotential Deposition of $\mathrm{Bi}^{3+}$ In $\mathrm{The}^{2}$ Presence of Coadsorbing $\mathrm{ClO}_{4}^{-}$on Gold”, Electrochimica Acta 47 (2002) 4113-4124.

[31] Halliwell, B. \& Gutteridge, J.M.C. (1999) Free Radical in Biology and Medicine. Oxford Science Publications, New York, NY.

[32] Henry J. Thompson, DNA Oxidation Products, Antioxidant Status, and Cancer Prevention, J. Nutr. 134: 3186S-3187S, 2004.

[33] Fridovich,I. (1989) Oxygen radicals from acetaldehyde. Free Radic. Biol. Med., 7, 557-558.

[34] Singh,N.P., Lai,H. and Khan,A. (1995) Ethanol-induced single strand DNA breaks in rat brain cells. Mutat. Res., 345, 191-196.

[35] Rajasinghe,H., Jayatilleke,E. and Shaw,S. (1990) DNA cleavage during ethanol metabolism: role of superoxide radicals and catalytic iron. Life Sci., 47, 807-814.

[36] Ewald,S.J. and Shao,H. (1993) Ethanol increases apoptotic cell death of thymocytes in vitro. Alcohol. Clin. Exp. Res., 17, 359-365.

[37] Moore,D.R., Reinke,L.A. and McCay,P.B. (1995) Metabolism of ethanol to 1-hydroxyethyl radicals in vivo: detection with intravenous administration of $\mu$-(4-pyridyl-1-oxide)-N-t-butylnitrone. Mol. Pharmacol., 47, 1224-1230.

[38] Gajewski, E., Rao, G., Nackerdien, Z., Dizdaroglu, M. (1990) Modification of DNA bases in mammalian chromatin by radiation-generated free radicals Biochemistry, 29, 7876-7882.

[39] Shibutani, S., Bodepudi, V., Johnson, F., Grollman, A.P. (1993) Translesional synthesis on DNA templates containing 8-oxo-7, 8-dihydrodeoxyadenosine Biochemistry, 32, 4615-4621.

[40] Wiederholt, C.J. and Greenberg, M.M. (2002) Fapy dG instructs Klenow exo(-) to misincorporate deoxyadenosine J. Am. Chem. Soc., 124, 7278-7279.

[41] Delaney, M.O., Wiederholt, C.J., Greenberg, M.M. (2002) Fapy•dA induces nucleotide misincorporation translesionally by a DNA polymerase Angew. Chem. Int. Ed. Engl., 41, 771-773.

[42] Nikita A. Kuznetsov, Vladimir V. Koval, Dmitry O. Zharkov, Georgy A. Nevinsky, Kenneth T. Douglas, and Olga S. Fedorova, Kinetics of substrate recognition and cleavage by human 8-oxoguanine-DNA glycosylase, Nucleic Acids Res., Jul 2005; 33: 3919 - 3931.

[43] Lindahl, T. (1974) An N-glycosidase from Escherichia coli that releases free uracil from DNA containing deaminated cytosine residues Proc. Natl. Acad. Sci. U.S.A, 71, 3649-3653.

[44] Stuart R.W. Bellamy, Kuakarun Krusong, and Geoff S. Baldwin, A rapid reaction analysis of uracil DNA glycosylase indicates an active mechanism of base flipping, Nucleic Acids Res., March 2007; 35: 1478 - 1487.

[45] Sandau, K., J. Pfeilschifter, and B. Brune. 1997. The balance between nitric oxide and superoxide determines apoptotic and necrotic death of rat mesangial cells. J. Immunol. 158:4938-4946.

[46] Yoshie, Y., and H. Ohshima. 1997. Nitric oxide synergistically enhances DNA strand breakage induced by polyhydroxyaromatic compounds, but inhibits that induced by the Fenton reaction. Arch. Biochem. Biophys. 342:13-21.

[47] Ken-ichiro Matsumoto and Hideo Utsumi, Development of Separable Electron Spin Resonance-Computed Tomography Imaging for Multiple Radical Species: An Application to · $\mathrm{OH}$ and ·NO, Biophys J, December 2000 , p. 3341-3349, Vol. 79, No. 6.

[48] Takeshita, K., H. Utsumi, and A. Hamada. 1993. Whole mouse measurement of paramagnetism-loss of nitroxide free radical in lung with an L-band ESR spectrometer. Biochem. Mol. Biol. Int. 29:17-24.

[49] Takeshita, K., A. Hamada, and H. Utsumi. 1999. Mechanisms related to reduction of radical in mouse lung using an L-band ESR spectrometer. Free Radic. Biol. Med. 26:951-960.

[50] Miura, Y., A. Hamada, and H. Utsumi. 1995. In vivo ESR studies of antioxidant activity on free radical reaction in living mice under oxidative stress. Free Radic. Res. 22:209-214.

[51] Phumala, N., T. Ide, and H. Utsumi. 1999. Noninvasive evaluation of in vivo free radical reactions catalyzed by iron using in vivo ESR spectroscopy. Free Radic. Biol. Med. 26:1209-1217.

[52] Matsumoto, K., K. Endo, and H. Utsumi. 2000. In vivo electron spin resonance assessment of decay constant of nitroxyl radical in selenium-deficient rat. Biol. Pharm. Bull. 23:641-644.

[53] Sano, H., M. Naruse, K. Matsumoto, T. Oi, and H. Utsumi. 2000. A new nitroxyl-probe with high retentivity in brain and its application to brain imaging. Free Radic. Biol. Med. 28:959-969. 